\title{
Herbert Stahl's proof of the BMV conjecture
}

\author{
Alexandre Eremenko*
}

July 17, 2018

\begin{abstract}
The paper contains a simplified version of Stahl's proof of a conjecture of Bessis, Moussa and Villani on the trace of matrices $A+t B$ with Hermitian $A$ and $B$.

MSC 2010 Class: 47A55, 30F10. Keywords: Hermitian matrices, perturbation theory, trace, Riemann surfaces.
\end{abstract}

This paper presents a simplified version of the proof of Herbert Stahl's theorem on the BMV conjecture [4. The proof preserves all main ideas of Stahl; the simplification consists in technical details.

Theorem. Let $A$ and $B$ be two $n \times n$ Hermitian matrices. Then the function

$$
f(t)=\operatorname{Tr} e^{A-t B}
$$

has a representation

$$
f(t)=\int_{b_{1}}^{b_{n}} e^{-s t} d \mu(s),
$$

where $\mu$ is a non-negative measure, $b_{1}$ and $b_{n}$ are the smallest and the largest eigenvalues of $B$.

If $B$ is positive semi-definite, it follows that $(-1)^{n} f^{(n)} \geq 0$. Such functions are called absolutely monotone. The result was conjectured in [1]. Two equivalent statements for positive semi-definite matrices $B$ are that the polynomial $t \mapsto \operatorname{Tr}(A+B t)^{p}, p \in \mathbf{N}$, has all non-negative coefficients, and that the function $t \mapsto \operatorname{Tr}(A+t B)^{-p}, p \geq 0$ is absolutely monotone, [2]. Before

\footnotetext{
*Supported by NSF.
} 
the work of Stahl, Theorem 1 was known for $2 \times 2$ matrices. The proof of Stahl, which is explained in these notes, is completely elementary: all needed tools were available in the middle of XIX century.

Without loss of generality, one can assume that $B$ is a diagonal matrix with eigenvalues $b_{n}>b_{n-1}>\ldots>b_{1}>0$. This is achieved by simultaneous conjugacy of $A$ and $B$, adding a scalar to $B$, and approximating the resulting $B$ with a matrix whose eigenvalues are distinct.

Now eigenvalues $\lambda$ of $A-t B$ are determined from the equation

$$
\operatorname{det}(\lambda I-A+t B)=0 .
$$

This determinant is a polynomial in two variables $t, \lambda$. We take $t$ out of the determinant, and denote $y=\lambda / t, x=1 / t$, then we obtain a polynomial equation of the form

$$
0=\operatorname{det}(y I+B-x A)=\prod_{j=1}^{n}\left(y+b_{j}-x a_{j, j}\right)+O\left(x^{2}\right),
$$

where $O\left(x^{2}\right)$ is a polynomial divisible by $x^{2}$.

This implies that there are $n$ holomorphic branches of the multivalued implicit function $\lambda(t)$ in a neighborhood of infinity, which satisfy

$$
\lambda_{j}(t)=-b_{j} t+a_{j, j}+O(1 / t), \quad t \rightarrow \infty,
$$

and all $\lambda_{j}$ are real on the real line. Moreover, each of these branches has an analytic continuation in a region containing the real line, according to Rellich's theorem [3, Thm XII.3]. The algebraic function $\lambda(t)$ is defined on a Riemann surface $S$ with $n$ sheets spread over the Riemann sphere. This Riemann surface is not necessarily connected. It has $n$ unramified sheets over a region that contains the real line and a neighborhood of infinity.

Special case. Suppose that $A$ is also diagonal, then the $O(1 / t)$ terms in (3) can be omitted, and we obtain

$$
f(t)=\sum_{j=1}^{n} e^{a_{j, j}} e^{-b_{j} t}=\int_{0}^{\infty} e^{-s t} \sum_{j=1}^{n} e^{a_{j, j}} \delta_{b_{j}}(s) d s .
$$

Thus $\mu$ is a discrete measure with positive atoms at the eigenvalues of $B$.

In the general case, the discrete component of $\mu$ is the same, and the continuous component is a positive function on $\left(b_{1}, b_{n}\right)$. 
Stahl figured out the following explicit expression for the density.

Proposition 1. The measure

$$
d \mu(s)=\left(\sum_{j=1}^{n} e^{a_{j, j}} \delta_{b_{j}}(s)+w(s)\right) d s
$$

where

$$
w(s)=-\sum_{j: b_{j}<s} \operatorname{res}_{\infty} e^{\lambda_{j}(\zeta)+s \zeta}=\frac{1}{2 \pi i} \sum_{j: b_{j}<s} \int_{C} e^{\lambda_{j}(\zeta)+s \zeta} d \zeta
$$

satisfies (11) and (2). Here $C$ is any circle centered at the origin, of sufficiently large radius, described counterclockwise.

First we give an heuristic argument which could be used to guess this formula. Inversion formula for the Laplace transform gives the density in the form

$$
\frac{1}{2 \pi i} \int_{L} f(\zeta) e^{s \zeta} d \zeta
$$

where $L$ is a vertical line sufficiently far to the right. For $|\zeta|$ large enough, the expression under the integral equals

$$
e^{s \zeta} f(\zeta)=\sum_{j=1}^{n} e^{\lambda_{j}(\zeta)+s \zeta}
$$

As $\lambda_{j}(\zeta)=-b_{j} \zeta+\ldots$, the summands for which $b_{j}>s$ are exponentially decreasing in the right half-lane, therefore, for these summands the line $L$ can be shifted to the right, and all these summands vanish. The rest of the summands exponentially decrease to the left, and for them, the contour can be bent to the left to obtain a circle $C$.

Of course one can give a rigorous justification of these arguments, but once the formula is guessed, it is easy to verify it directly, and we reproduce Stahl's argument.

Lemma 1. For every s, we have

$$
\sum_{j=1}^{n} \int_{C} e^{\lambda_{j}(\zeta)+s \zeta} d \zeta=0
$$

Indeed, this is an integral of an entire function over a closed contour. 
It follows that the density $w$ defined by (6) is zero for $s>b_{n}$, and it is evidently zero for $s<b_{1}$.

Proof of Proposition 1. We compute the Laplace transform of the density $w$ defined by (6) $)$.

$$
\int_{0}^{\infty} e^{-s t} w(s) d s=\sum_{k=1}^{n-1} \int_{b_{k}}^{b_{k+1}} e^{-t s} w(s) d s=: \sum_{k=1}^{n-1} I_{k}(t) .
$$

We fix $t>0$ and deform the contour $C$ in (6) so that the positive ray is outside $C$. This is possible to do because all $\lambda_{j}$ are holomorphic in a region containing the real line and $C$. Thus $t$ is outside of the deformed contour $C^{\prime}$. According to (6), we have

$$
I_{k}(t)=\int_{b_{k}}^{b_{k+1}} \sum_{j=1}^{k} \frac{1}{2 \pi i} \int_{C^{\prime}} e^{\lambda_{j}(\zeta)+s(\zeta-t)} d \zeta d s .
$$

By changing the order of integration and the order of summation, we obtain

$$
\begin{aligned}
\sum_{k=1}^{n-1} I_{k}(t) & =\sum_{j=1}^{n-1} \frac{1}{2 \pi i} \int_{C^{\prime}} e^{\lambda_{j}(\zeta)} \int_{b_{j}}^{b_{n}} e^{s(\zeta-t)} d s d \zeta \\
& =\sum_{j=1}^{n-1} \frac{1}{2 \pi i} \int_{C^{\prime}} e^{\lambda_{j}(\zeta)}\left(e^{b_{n}(\zeta-t)}-e^{b_{j}(\zeta-t)}\right) \frac{d \zeta}{\zeta-t}
\end{aligned}
$$

The last expression is transformed using Cauchy's formula and the fact that $t$ is outside $C^{\prime}$. We have

$$
\sum_{j=1}^{n} \int_{C^{\prime}} e^{\lambda_{j}(\zeta)} e^{b_{n}(\zeta-t)} \frac{d \zeta}{\zeta-t}=0
$$

similarly to Lemma 1 , so

$$
\sum_{k=1}^{n-1} I_{k}(t)=-\sum_{j=1}^{n} \frac{1}{2 \pi i} \int_{C^{\prime}} e^{\lambda_{j}(\zeta)+b_{j}(\zeta-t)} \frac{d \zeta}{\zeta-t} .
$$

Using (3), we write $\lambda_{j}(\zeta)=-b_{j} \zeta+a_{j, j}+r_{j}(\zeta)$, where $r_{j}(\infty)=0$, and apply Cauchy's formula again. We obtain for every $j$ :

$$
\begin{aligned}
& -\frac{1}{2 \pi i} \int_{C^{\prime}} e^{\lambda_{j}(\zeta)+b_{j}(\zeta-t)} \frac{d \zeta}{\zeta-t}=-\frac{e^{-b_{j} t+a_{j, j}}}{2 \pi i} \int_{C^{\prime}} e^{r_{j}(\zeta)} \frac{d \zeta}{\zeta-t} \\
& =e^{-b_{j} t+a_{j, j}}\left(e^{r_{j}(t)}-1\right)=e^{\lambda_{j}(t)}-e^{-b_{j} t+a_{j, j}}
\end{aligned}
$$


Adding these expressions for $j=1 \ldots n$ and comparing with (5) and the second equation in (4), we obtain Proposition 1.

It remains to prove that (6) is non-negative for every $s$. Let us fix $s$ and $k$ so that $b_{k}<s<b_{k+1}$. The idea of Stahl, is to replace the contour of integration in (6) by an ingeniously chosen homologous contour, on which the integral is non-negative simply because the integrand is non-negative.

We recall that $S$ is a (possibly disconnected) Riemann surface spread over the $\zeta$-sphere. We denote a generic point of $S$ by $p$, and let $\pi: S \rightarrow \overline{\mathbf{C}}$ be the projection to $\zeta$-plane. Then $\lambda$ is a meromorphic function on $S$ whose all poles are simple and lay over $\zeta=\infty$.

Asymptotic expressions (3) imply that there exists $R>0$ such that for all $j \leq k$ the functions

$$
\lambda_{j}(\zeta)+s \zeta=\left(s-b_{j}\right) \zeta+\ldots
$$

are holomorphic for $|\zeta|>R$, real on the real line and have strictly positive derivatives for $\zeta>R$ and $\zeta<-R$, while for $j>k$ they have strictly negative derivatives. By increasing $R$, if necessary, we achieve that for $|\zeta|>R / 2$ and $j \leq k$, we have that $\operatorname{Im}\left(\lambda_{j}(\zeta)+s \zeta\right)$ has the same $\operatorname{sign}$ as $\operatorname{Im} \zeta$. And for $|\zeta|>R / 2$ and $j>k, \operatorname{Im}\left(\lambda_{j}(\zeta)+s \zeta\right)$ has the opposite sign from $\operatorname{Im} \zeta$.

The surface $S$ has an anti-conformal involution, induced by complex conjugation. The set of fixed points of this involution consists of $n$ curves, $\pi$-preimages of the real line. These curves break $S$ into two halves $S^{+}$and $S^{-}$which are mapped onto each other by the involution. Projections of these halves are the upper and lower half-planes.

We set $C=\{\zeta:|\zeta|=R\}$ in (6), where $R$ was just chosen.

Consider the open sets

$$
\begin{aligned}
& D^{+}:=\{p \in S:|\pi(p)|<R, \operatorname{Im} \pi(p)>0, \operatorname{Im}(\lambda(p)+s \pi(p))>0\}, \\
& D^{-}:=\{p \in S:|\pi(p)|<R, \operatorname{Im} \pi(p)<0, \operatorname{Im}(\lambda(p)+s \pi(p))<0\},
\end{aligned}
$$

and

$$
D=\operatorname{int}\left(\overline{D^{+}} \cup \overline{D^{-}}\right) .
$$

The set $\{p \in S:|\pi(p)|=R\}$ consists of $n$ disjoint circles $C_{j} \subset S$ which we label according to the branches of $\lambda_{j}$ in (7), so that $\lambda=\lambda_{j}$ on $C_{j}$. According to the paragraph after (7), the circles $C_{j}$ with $j \leq k$ belong to $\partial D$ while the $C_{j}$ with $j>k$ are disjoint from $\bar{D}$. 
Let $D_{1}$ be a component of $D$ whose boundary contains some circles $C_{j} 1$ We are going to prove that

$$
\sum_{j: C_{j} \subset \partial D_{1}} \int_{C_{j}} e^{\lambda(p)+s \pi(p)} d \pi(p)>0,
$$

where the circles are oriented counterclockwise, which agrees with their orientation as part of $\partial D$. Adding these relations over all components of $D$ will prove the theorem. Indeed, each circle $C_{j}$ with $j \leq k$ belongs to the boundary of exactly one component of $D$, and circles $C_{j}$ with $j>k$ do not belong to the boundary of $D$.

Each component $D_{1}$ of $D$ is a Riemann surface of finite type, whose boundary consists of several curves parametrized by circles. This parametrization is piecewise smooth, but may be neither smooth nor injective. We call these curves the boundary curves of $D_{1}$. Our choice of $R$ guarantees that the part of the boundary of $D_{1}$ that projects in $C$ is exactly the chain on which the integration is performed in (8) . Consider the rest of the boundary $\partial D_{1}$ which projects into $|\zeta|<R$.

Lemma 2. No boundary curve of $D$ over $\{\zeta:|\zeta|<R\}$ can project into the open upper or lower half-plane.

Indeed suppose that $\gamma$ is a boundary curve whose projection does not intersect the real axis. It is oriented in the standard way, so that $D$ is on the left. Suppose without loss of generality that $\gamma$ projects to the upper half-plane. Let $g(p)=\lambda(p)+s \pi(p)$. As $\operatorname{Im} g>0$ in $D^{+}$, and $\operatorname{Im} g=0$ on $\gamma$, we conclude that the normal derivative of $\operatorname{Im} g$ has constant sign on $\gamma$. Then by the Cauchy-Riemann equations, the tangential derivative of $\operatorname{Re} g$ along $\gamma$ is of constant sign, which is impossible because $\gamma$ is a closed curve, and $\operatorname{Re} g$ is single valued on $\gamma$.

Thus every boundary curve of $D_{1}$ intersects the real line. Let $\gamma$ be a boundary curve of $D_{1}$ which projects into $\{\zeta:|\zeta|<R\}$. By Lemma $2, \gamma$ is mapped into itself by the involution, so it consists of two symmetric pieces: one piece $\gamma^{+}$projects in the upper half-plane, another $\gamma^{-}$to the lower halfplane. At all endpoints $p$ of $\gamma^{+}$or $\gamma^{-}$we have $\Im \pi(p)=0$. We have

$$
e^{g}=e^{\operatorname{Re} g+i \operatorname{Im} g}=e^{\operatorname{Re} g}(\cos (\operatorname{Im} g)+i \sin (\operatorname{Im} g)) .
$$

\footnotetext{
${ }^{1}$ One can prove using the maximum principle that every component of $D$ has some $C_{j}$ on the boundary, but we are not using this fact.
} 
Since $\operatorname{Im} g=0$ on $\gamma$, and $\operatorname{Re} g$ is increasing, we conclude that $\phi(t):=e^{g(\gamma(t))}$ is real and increasing function of the natural parameter $t$ on $\gamma^{+}$. Thus

$$
\begin{aligned}
\frac{1}{2 \pi i} \int_{\gamma} e^{g(p)} d \pi(p) & =\frac{1}{2 \pi i}\left(\int_{\gamma^{+}}+\int_{\gamma^{-}}\right) \phi(t)(d \xi(t)+i d \eta(t)) \\
& =\frac{1}{\pi} \int_{\gamma^{+}} \phi(t) d \eta(t)=-\frac{1}{\pi} \int_{\gamma^{+}} \eta(t) d \phi(t)<0,
\end{aligned}
$$

where we integrated by parts using $\eta(t)=0$ on the endpoints of $\gamma^{+}$.

As the integral of the holomorphic 1-form over the boundary equals zero,

$$
\int_{\partial D_{1}} e^{g} d \pi=0
$$

by Cauchy's theorem, the contribution to the integral from the part of $\partial D_{1}$ which projects to $\{\zeta:|\zeta|<R\}$ is the negative of the contribution of the part of $\partial D_{1}$ over $C$. This completes the proof of (8) and of Theorem 1.

\section{References}

[1] D. Bessis, P. Moussa and M. Villani, Monotonic converging variational approximations to the functional integrals in quantum statistical mechanics, J. Math. Phys., 16 (1975) 2318-2385.

[2] E. Lieb and R. Steiringer, Equivalent forms of the Bessis-Moussa-Villani conjecture, J. Stat. Phys., 115 (2004) 185-190.

[3] M. Reed, B. Simon, Methods of mathematical physics, vol. IV, Analysis of operators, Acad. Press, NY 1978.

[4] H. Stahl, Proof of the BMV conjecture, Acta Math., 211, 2 (2013) 255290.

Purdue University

eremenko@math.purdue.edu 